\title{
Utilisations digestive et métabolique comparées de la fève, de la lentille et du pois chiche chez le rat
}

\author{
E Combe, T Achi, R Pion
}

\author{
avec la collaboration technique de MC Valluy, ML Houlier, M Sallas, A Selle \\ INRA, laboratoire d'étude du métabolisme azoté, 63122 Saint-Genès-Champanelle, France
}

(Reçu le 6 avril 1990; accepté le 28 août 1991)

\begin{abstract}
Résumé - Dans une étude effectuée chez le rat, les graines crues de fève, lentille ou pois chiche ont été utilisées comme seule source protéique. Le régime (12\% MA) a été calculé de façon à satisfaire les besoins de la croissance. Le CUDa de l'azote est respectivement de $72-75-76 \%$ dans le cas des lots fève - lentille - pois chiche, mais le CUDa de certains acides aminés indispensables est nettement plus faible : $52-51-71$ pour la méthionine; $60-39-78$ pour la cystine; $73-71-$ 75 pour la valine alors que le CUDa de l'arginine est toujours plus élevé $87-87-82$ dans le cas de la fève, de la lentille et du pois chiche respectivement. Les vitesses de croissance $(6,1 \mathrm{a} 9,1 \mathrm{~g} / \mathrm{j})$ et les indices de consommation $(2,1$ à 2,5$)$ sont satisfaisants mais l'étude des concentrations d'acides aminés libres du sang et du muscle, en relation avec les quantités ingérées digestibles, permet de déceler une disparité de la disponibilité des acides aminés apportés par les graines des 3 légumineuses. Nous en concluons que la thréonine du pois chiche, l'arginine et la lysine de la fève ainsi que la méthionine des 3 graines ne semblent que partiellement disponibles.
\end{abstract}

fève / lentille / pois chiche / acide aminé / digestibilité / utilisation métabolique

Summary - Compared metabolic and digestive utilisations of faba bean, lentil and chick pea. Faba bean, lentil or chick-pea raw seeds were used as the only protein food in the rat. The balanced diet was designed to suit growth requirements. Nitrogen apparent digestibility coefficient (CUDa) was $72 \%$ in the faba bean, $75 \%$ in the lentil, $76 \%$ in the chick-pea groups respectively, but the CUDa of some essential amino acids were much lower : $52-51-71 \%$ for methionine, $60-39-78 \%$ for cystine, $73-71-75 \%$ for valine, while arginine CUDa values $(87-87-82)$ were higher than all other AA CUDa values. Growth rate $(8.9-6.7-9.1 \mathrm{~g} / d)$ and food intake index $(2.1-2.5-2.2)$ were satisfactory. Blood and muscle free amino acid concentrations related to digestible intake showed that methionine from the 3 legumes, threonine from chick-pea, arginine and lysine from faba bean were not fully available.

fababean / lentil / chick-pea / amino acid / digestibility / availabllity 


\section{INTRODUCTION}

Les graines des légumineuses présentent un intérêt nutritionnel original : elles sont riches en protéines et apportent en même temps des sucres à digestion lente et des fibres. Leur utilisation en alimentation animale et humaine a cependant été freinée par la présence de facteurs glucidiques ou antinutritionnels, identifiés en quantité variable selon la partie anatomique et l'état de maturation de la graine. Ces facteurs peuvent en effet entraîner des modifications dans les phénomènes de la digestion enzymatique ou microbienne (MendèsPereira et al, 1977; Carré et Leclercq, 1985; Newman et al, 1987; Combe et al, 1988; Lacassagne et al, 1988).

La fève (Vicia faba), la lentille (Lens culinaris ou Lens esculenta) et le pois chiche (Cicer arietinum) sont les espèces les plus utilisées parmi les légumes secs dans les pays tempérés et méditerranéens. Leur composition globale varie selon les espèces, les variétés, les origines géographiques et les conditions de culture, de récolte et de stockage. Elles apportent près de 1,7 MJ pour $100 \mathrm{~g}$ de graines sèches (cf tableau 1) avec une très faible teneur en lipides compensée par leur teneur importante en glucides $(66-74 \mathrm{~g} / 100 \mathrm{~g})$ et en protéines $(20-30 \mathrm{~g} / 100 \mathrm{~g})$. Leur teneur en fibre est de $4,3 \%$ pour la lentille; elle varie de 4,48 à $8,5 \mathrm{~g} \%$ pour la fève et de 2,8 à 7,4 pour le pois chiche selon les auteurs. L'apport minéral des graines n'est pas négligeable $(2,4-3,8 \%)$ notamment en magnésium et oligoéléments.

Les tables de Paul et Southgate (1978) montrent que les graines de ces 3 légumineuses sont très riches en lysine, arginine et acides aminés dicarboxyliques, mais carencées en acides aminés soufrés ainsi qu'en tryptophane et thréonine quand on les compare aux besoins en acides aminés indispensables du rat en croissance.
C'est pourquoi, nous avons voulu étudier comparativement l'utilisation digestive et métabolique des acides aminés apportés par les graines de fèves, lentilles ou pois chiches. Dans ce but, nous avons utilisé les graines entières à l'état cru comme seule source protéique dans la ration alimentaire du rat en croissance. Après avoir déterminé dans chaque lot la digestibilité fécale apparente pour chaque acide aminé, nous avons recherché une relation entre les pools d'acides aminés libres du sang et du muscle, et les quantités d'acides aminés digestibles ingérées.

\section{MATÉRIEL ET MÉTHODES}

\section{Régimes alimentaires}

Les graines utilisées provenaient de l'Institut de développement des grandes cultures de Constantine : il s'agissait de fève (Vicia faba) variété Séville récoltée en zone sublittoraje, de lentille (Lens esculenta) variété Petite Blonde de Dahra récoltée en zone des hauts plateaux et de pois chiche (Cicer arietinum) variété Rabat 9 récoltée en zone sublittorale. Avant d'être incorporées au régime, elles ont été broyées et passées sur une grille de $0,8 \mathrm{~mm}$.

\section{Expérience de digestibilité}

Les régimes sont calculés sur la base de la composition des graines, afin d'apporter $12 \%$ de matière azotée ( $\mathrm{MA}=\mathrm{N} \times 6,25$ ) et $18,8 \mathrm{MJ} / \mathrm{kg}$ de matière sèche (MS). La supplémentation en tryptophane et en acides aminés soufrés, thréorine, leucine et isoleucine (tableau II) de l'apport protéique des graines a été calculée en fonction de sa composition en acides aminés indispensables de taçon à ce que l'ingéré corresponde aux besoins du rat en croissance. Les proportions de la ration énergétique apportées par les graines de fève, lentille et pois chiche sont respectivement de 45,40 et $48 \%$ dans ce premier essai. L'apport énergétique des graines est complété par de l'amidon de blé et par de l'huile d'arachide. De même, le complément minéral 
Tableau I. Composition globale des graines de fève, lentille et pois chiche (pour $100 \mathrm{~g} \mathrm{secs).}$

\begin{tabular}{|c|c|c|c|}
\hline & $\begin{array}{c}\text { Fève } \\
\text { Vicia faba }\end{array}$ & $\begin{array}{c}\text { Lentille } \\
\text { Lens esculenta }\end{array}$ & $\begin{array}{c}\text { Pois chiche } \\
\text { Cicer arietinium }\end{array}$ \\
\hline Énergie brute (MJ) & $1,6^{a}$ & $\begin{array}{l}1,6^{a} \\
1,4^{b} \\
1,7^{c}\end{array}$ & $\begin{array}{l}1,7^{a} \\
1,5^{b}\end{array}$ \\
\hline Glucides (g) & $66^{a}$ & $68-74^{a}$ & $69^{a}$ \\
\hline $\begin{array}{l}\text { Protéines }(g) \\
(N \times 6,25)\end{array}$ & $\begin{array}{l}29^{a} \\
25,9^{d} \\
28,4^{\theta} \\
25^{\dagger} \\
329^{9} \\
22,8^{h}\end{array}$ & $\begin{array}{l}23-27^{a} \\
28,6^{c} \\
25^{\mathrm{i}} \\
28,1^{1} \\
26,9^{k} \\
24,2^{\prime}\end{array}$ & $\begin{array}{l}21^{a} \\
20,1^{1} \\
21-24^{m}\end{array}$ \\
\hline Lipides (g) & $\begin{array}{l}1,4^{a} \\
2,1^{d} \\
1,91^{e} \\
1,2^{f} \\
0,99\end{array}$ & $\begin{array}{l}1,3-0,7^{a} \\
0,7^{c}\end{array}$ & $\begin{array}{l}4,1-6,2^{\mathrm{a}} \\
5,4^{\mathrm{m}}\end{array}$ \\
\hline Fibres (g) & $\begin{array}{l}5,9^{a} \\
4,48^{\circ} \\
5-6,9^{\prime} \\
8,5^{9}\end{array}$ & $\begin{array}{l}4,3^{a} \\
4,4^{c}\end{array}$ & $7,4-2,8^{a}$ \\
\hline Cendres $(g)$ & $\begin{array}{l}3,6^{\mathrm{a}, \mathrm{g}} \\
3,4^{\mathrm{d}} \\
3,84^{\mathrm{\theta}} \\
2,7-3,6^{\mathrm{f}}\end{array}$ & $\begin{array}{l}3,2-2,4^{a} \\
3,1^{c} \\
3,6 j\end{array}$ & $\begin{array}{l}3,5-3,5^{a} \\
3,34^{!}\end{array}$ \\
\hline
\end{tabular}

a : Duke (1981); b : Gupta (1982); $\mathrm{:} \mathrm{Bhatty} \mathrm{et} \mathrm{al} \mathrm{(1976);} \mathrm{d}$ : Colonna et al (1981); $\theta$ : Tanusi et al (1972); $\mathrm{f}$ : Askar (1986); g : Marquardt et al (1975); h : Pion (1973); i : El Shobaki et Saleh (1986); j : Jarrige (1988); $k$ : Shekib et al (1986); I: Kandé (1967); $m$ : Khanvilkar et al (1981).

apporte $\mathrm{Ca}, \mathrm{P}, \mathrm{Mg}$ et $\mathrm{Na}$. Les vitamines et les oligo-éléments proviennent d'un mélange standard (Combe et al, 1988). Les constituants apportés sous forme de poudre sont mélangés en milieu aqueux à température ambiante. Les régimes homogénéisés sont conservés sous forme semi-liquide au froid $\left(4^{\circ} \mathrm{C}\right)$ au maximum $3 \mathrm{j}$ et distribués ad libitum, quotidiennement.

\section{Expérience de croissance}

Dans ce deuxième essai, les régimes ont été recalculés à partir de nos propres résultats d'analyse de la composition en acides aminés de ces graines (tableau III) et de la valeur des coeffi- cients d'utilisation digestive apparente obtenus dans le premier essai. Les taux de MA des rations deviennent $19 ; 15$ et $17 \%$ ce qui correspond finalement à des taux de MA digestible de 13,$3 ; 11,7$ et $13 \%$ respectivement pour les lots fève, lentille et pois chiche. Les proportions de la ration énergétique fournies par les graines de fève, lentille, pois chiche deviennent respectivement 62,51 et $75 \%$.

\section{Animaux}

Les rats mâles en croissance de race Sprague Dawley reçus à l'âge de 4 semaines et placés 
Tableau II. Composition des régimes ( $\mathrm{g} / \mathrm{kg} \mathrm{sec}$ ).

\begin{tabular}{|c|c|c|c|c|c|c|}
\hline & & ience de c & ligestibilité & & ience de & oissance \\
\hline & Fève & Lentille & Pois chiche & Fève & Lentille & Pois chiche \\
\hline Graines & 521 & 462 & 532 & 732 & 599 & 789 \\
\hline Amidon de blé & 270 & 292 & 226 & 115 & 117 & 117 \\
\hline Agar-Agar & 25 & 25 & 24 & 25 & 26 & 25 \\
\hline Huile d'arachide & 184 & 221 & 218 & 128 & 133 & 51 \\
\hline Supplémentation & & & & & & \\
\hline $\mathrm{Ca}$ & 9,49 & 9,82 & 9,20 & 9,30 & 9,77 & 8,90 \\
\hline $\mathbf{P}$ & 5,31 & 5,91 & 5,29 & 4,62 & 5,60 & 4,67 \\
\hline $\mathrm{Mg}$ & 0,13 & 0,65 & 0,09 & 0,00 & 0,55 & 0,00 \\
\hline $\mathrm{Na}$ & 1,86 & 1,84 & 1,77 & 1,79 & 1,79 & 1,69 \\
\hline Supplémentation & & & & & & \\
\hline Thr & 0,00 & 0,27 & 0,26 & 0,00 & 0,00 & 0,00 \\
\hline Cys & 1,42 & 1,76 & 1,12 & 0,70 & 1,28 & 0,00 \\
\hline Met & 1,31 & 2,05 & 1,46 & 1,70 & 1,44 & 0,13 \\
\hline$\| e$ & 0,14 & 0,00 & 0,00 & 0,00 & 0,00 & 0,00 \\
\hline Leu & 0,00 & 0,00 & 1,29 & 0,00 & 0,00 & 0,00 \\
\hline Trp & 0,20 & 0,36 & 0,53 & 0,00 & 0,05 & 0,18 \\
\hline
\end{tabular}

AAl : acides aminés indispensables.

en cage grillagée dans une raterie éclairée de 7 à $19 \mathrm{~h}$ et maintenue à $22{ }^{\circ} \mathrm{C}$ et à $60 \%$ d'humidité relative reçoivent un aliment standard Sanders $(20 \% \mathrm{MA})$ jusqu'au poids moyen de $70 \mathrm{~g}$ ils sont alors répartis en lots homogènes et installés dans les cages griliagées individuelles munies éventuellement d'un dispositif permettant la collecte séparée de l'urine et des fèces.

L'expérience de digestibilité est réalisée sur 15 rats, répartis en 3 lots de 5, et recevant chacun l'un des régimes expérimentaux (fève, lentille ou pois chiche), pendant 13 j consécutifs : 8 j d'habituation et 5 j de mesure des quantités ingérées et excrétées. Les matières sèches ingérées (MSI) sont mesurées quotidiennement. Les fèces et les urines sont collectées quantitativement chaque jour et stockées à $-15^{\circ} \mathrm{C}$. Les quantités excrétées individuellement sont déterminées sur le total recueilli au cours de la période de mesure.

L'expérience de croissance est effectuée sur 45 rats répartis en 3 lots de 15 . Chaque lot reçoit l'un des 3 régimes pendant $17 \mathrm{j}: 7 \mathrm{j}$ d'habituation et $10 \mathrm{j}$ de mesure de la consommation
(MSI déterminée tous les jours) et de la croissance (poids initial $=100 \mathrm{~g} \pm 7$; contrôle tous les 2 jours). A la fin de la période expérimentale, les rats de chaque lot sont anesthésiés à l'éther, le sang carotidien est recueilli directement dans 7 fois son volume d'éthanol froid à $80 \%$ et pesé. Les muscles des membres inférieurs sont disséqués, pesés, congelés immédiatement dans l'azote liquide. Les échantillons individuels de sang et de muscle sont regroupés par lots et stockés à $-20^{\circ} \mathrm{C}$ en vue de la préparation pour l'analyse des acides aminés libres.

\section{Préparation des échantillons et analyses}

La matière sèche fécale (MSF) est déterminée pour chaque rat par pesée avant et après lyophilisation des fèces récoltées. Les fèces lyophilisées sont alors regroupées par lot, broyées et homogénéisées. Les différents dosages sont effectués sur des quantités aliquotes d'un poids 
Tableau III. Composition globale et composition en acides aminés ( $g / 16 \mathrm{~g}$ d'N) de la fève, de la lentille et du pois chiche.

\begin{tabular}{lccc}
\hline & $\begin{array}{c}\text { Fève } \\
\text { Séville }\end{array}$ & $\begin{array}{c}\text { Lentille } \\
\text { PB Dahra }\end{array}$ & $\begin{array}{c}\text { Pois chiche } \\
\text { Rabat } 9\end{array}$ \\
& & & \\
MS (\%) & 92,49 & 90,95 & 91,20 \\
MO brute (\%) & 95,99 & 97,35 & 96,68 \\
Cellulose \% & 9,7 & 3,5 & 3,8 \\
Énergie brute (MJ/100 g) & 1,66 & 1,66 & 1,76 \\
MA (\%MS) & 25,90 & 25,98 & 21,74 \\
& & & \\
Asp & 10,75 & 11,50 & 11,90 \\
Thr & 3,40 & 3,65 & 3,80 \\
Ser & 4,80 & 5,05 & 5,35 \\
Glu & 15,20 & 15,85 & 16,35 \\
Pro & 3,65 & 3,90 & 4,65 \\
Gly & 4,25 & 4,05 & 4,25 \\
Ala & 4,30 & 3,95 & 4,45 \\
Val & 4,55 & 4,60 & 2,30 \\
Cys & 1,40 & 1,10 & 1,70 \\
Met & 0,90 & 1,05 & 4,40 \\
lleu & 4,05 & 4,05 & 7,30 \\
Leu & 6,65 & 6,80 & 3,35 \\
Tyr & 3,55 & 3,20 & 5,75 \\
Phe & 3,60 & 4,30 & 7,30 \\
Lys & 6,55 & 6,50 & 2,95 \\
His & 2,45 & 2,40 & 10,15 \\
Arg & 9,25 & 8,85 & \\
AAl/TAA (\%) & & & 53,33 \\
Bilan azoté & 51,81 & 51,17 & 90,08 \\
& & 81,01 & \\
\hline & 80,86 & & \\
\hline
\end{tabular}

MS : matière sèche; MA : matière azotée $=\mathrm{N} \times 6,25 ;$ MO : matière organique; $A A 1$ : acides aminés indispensables; TAA : total des acides aminés. Bilan azotée $=\% \mathrm{~N}$ des acides aminés/ $\mathrm{N}$ Kjeldahl.

suffisant pour permettre une détermination aisée des différents composés. Le dosage d'azote des aliments, des fèces et des urines est réalisé par la méthode de Kjeldahl (Kjeltec Auto-Analyser 1030 , Tecator) et les acides aminés totaux des aliments et des fèces sont dosés après hydrolyse acide $(\mathrm{HCl} 5,5 \mathrm{~N})$ de 24 et $48 \mathrm{~h}$ et chromatographie sur résine échangeuse de cations (Moore et al, 1958). On utilise une oxydation performique préalable à l'hydrolyse, pour le dosage de la méthionine et de la cystine. Le tryptophane n'a pas été dosé. L'énergie brute to- tale est déterminée à l'aide d'un calorimètre (Gallenkamp Adiabatic). La cellulose (insoluble cellulosique) a été dosée par la méthode Weende (Fibertec, Técator).

Du fait de la complexité et de la longueur des opérations de préparation des échantillons pour le passage en chromatographie quantitative sur colonne, les acides aminés libres sont déterminés sur les échantillons individuels regroupés pour chaque lot. Les acides aminés libres du sang sont extraits par l'éthanol $84 \%$ à froid. Les 
extraits éthanoliques obtenus sont purifiés par passage sur une résine échangeuse de cations Amberlite IR 120 (polystyrène sulfonique sous forme $\mathrm{H}^{+}$); ceux des muscles sont extraits par l'acide picrique à $1 \%$. Les divers composés réagissant à la ninhydrine sont séparés et dosés selon la méthode décrite par Pawlak et Pion (1968). Le coefficient de variation des teneurs en acides aminés obtenus sur 5 rats ayant reçu le même régime témoin caséine (ou lentilles crues) est de $17 \%$ (14\%) pour le total des acides aminés dosés. II varie selon l'acide aminé de $10 \%(12 \%)$ pour l'arginine à $27 \%$ (25\%) pour la thréonine.

Les teneurs en $P$ des graines ont été déterminées par colorimétrie, celles en $\mathrm{Ca}, \mathrm{K}, \mathrm{Na}$, par photométrie de flamme. Les teneurs en $\mathrm{Mg}$ ainsi qu'en $\mathrm{B}, \mathrm{Cu}, \mathrm{Fe}, \mathrm{Mn}$ et $\mathrm{Zn}$ ont été dosées par absorption atomique. L'acide tanique a été dosé par la méthode de Folin-Denis (colorimétrie), rapportée par Tempel (1982).

\section{RÉSULTATS}

\section{Composition des graines de fève, lentille et pois chiche}

Le tableau III montre que l'apport énergétique du pois chiche est à peine plus élevé que celui de la fève ou de la lentille. En outre, la teneur en cellulose de la fève est 2,6 fois plus élevée que celle de la lentille ou du pois chiche.

Les teneurs en MA $(N \times 6,25)$ de la fève et de la lentille atteignent presque $26 \mathrm{~g} /$ $100 \mathrm{~g}$ alors que celle du pois chiche est plus faible $(-16 \%)$. Cependant, le pois chiche $\epsilon$ st plus riche en acides aminés et la proportion des acides aminés indispensables y est un peu plus importante que dans le cas de la fève et de la lentille.

La comparaison de l'équilibre des acides aminés indispensables (AAl) correspondant aux besoins du rat en croissance à celui apporté par les graines des 3 légumineuses étudiées fait apparaître que les proportions d'arginine, d'histidine et même de tyrosine dans le cas de la fève, sont supérieures aux besoins. La proportion d'arginine présente dans les graines de fève, lentille et pois chiche est 2 fois supérieure à celle exprimée par les besoins. Si on exclut l'arginine de la comparaison, seules les proportions des acides aminés soufrés apparaissent nettement insuffisantes dans les 3 légumineuses. Ainsi la proportion de cystine s'écarte de 32, 48 et $16 \%$ (celle de méthionine s'écarte de 69,48 et $32 \%$ ) par rapport à l'équilibre correspondant aux besoins, dans le cas de la fève de la lentille et du poids chiche, respectivement.

Le tableau IV montre que les graines de fève, lentille et pois chiche étudiées sont riches en $\mathrm{P}$ (bien que sous forme phytique à 60-70\%) et relativement riches en $\mathrm{Mg}$; si la lentille a les plus faibles concentrations en ces minéraux, elle contient 2 fois plus de $\mathrm{Fe}$ et de $\mathrm{Cu}$, que la fève et le pois chiche. Les teneurs en acide tanique de la fève et de la lentille sont 2,8 fois plus élevées que celles du pois chiche.

\section{Utilisation digestive des trois légumineuses}

Le coefficient d'utilisation digestive apparente (CUDa) de la matière sèche est significativement $(P<0,01)$ plus faible $(-7 \%)$ pour la fève que pour la lentille et le pois chiche (tableau V). Le CUDa de l'énergie est plus faible pour la fève $(-8 \%)$ que pour la lentille $(-2,7 \%)$ et le pois chiche. Le CUDa des matières azotées est plus faible pour la fève $(-7 \%)$ que pour la lentille et le pois chiche.

Le CUDa du total des acides aminés (TAA) et des acides aminés indispensables (AAl) est plus élevé (+ $11 \%$ ) dans le cas du lot pois chiche que dans le cas des lots fève et lentille. 
Tableau IV. Composition en minéraux et en acide tanique de la fève, de la lentille et du pois chiche.

\begin{tabular}{lrcc}
\hline & Fève & Lentille & Pois chiche \\
\hline P total $(\mathrm{g} / \mathrm{kg})$ & 5,5 & 3,8 & \\
$\mathrm{Ca}$ total $(\mathrm{g} / \mathrm{kg})$ & 2,1 & 0,9 & 4,6 \\
$\mathrm{Mg}(\mathrm{g} / \mathrm{kg})$ & 1,4 & 1,0 & 1,2 \\
$\mathrm{~K}(\mathrm{~g} / \mathrm{kg})$ & 13,7 & 9,0 & 1,3 \\
$\mathrm{Na}(\mathrm{g} / \mathrm{kg})$ & 0,5 & 0,4 & 0,4 \\
& & & \\
$\mathrm{~B}$ total $(\mathrm{mg} / \mathrm{kg})$ & 13,2 & 8,6 & 12,4 \\
Cu total $(\mathrm{mg} / \mathrm{kg})$ & 15,0 & 126,0 & 10,0 \\
Fe total $(\mathrm{mg} / \mathrm{kg})$ & 68,0 & 14,0 & 61,0 \\
Mn total $(\mathrm{mg} / \mathrm{kg})$ & 14,0 & 29,0 & 3,0 \\
Zn total $(\mathrm{mg} / \mathrm{kg})$ & 27,0 & & 33,0 \\
& & & \\
Acide tanique $(\mathrm{g} / \mathrm{kg})$ & $6,98 \pm 0,21$ & $7,22 \pm 2,20$ & $2,55 \pm 0,57$ \\
Moyenne \pm écart type & & & \\
\hline
\end{tabular}

Tableau V. Utilisation digestive apparente (CUDa \%) (1) de la matière sèche (2), de l'énergie (3) de l'azote et des acides aminés (TAA et AAl).

\section{Fòve Lentille Poischiche}

\begin{tabular}{lccc} 
MS & $78,90 \pm 0,97^{*}$ & $84,03 \pm 0,84$ & $85,92 \pm 2,16$ \\
Énergie & 81,00 & 85,60 & 88,00 \\
Azote & 70,50 & 75,00 & 75,60 \\
TAA & 70,51 & 72,06 & 80,01 \\
AAI & 72,74 & 71,06 & 79,70 \\
\hline
\end{tabular}

(1) : CDUa = $100(I-F) / l ; \mid=$ Ingéré; $F=$ Excrété dans les fèces pour la même période; (2) : moyenne \pm écart type. " : significativement different de ceux de la lentille et du pois chiche $(P<0,01)$; (3) : l'énergie, "azote et les $A A$ ont été déterminés sur les echantillons regroupés.

Si l'on considère la figure 1 , il est clair que ni le CUDa de l'azote (MA) ni le CUDa des $A A l$ ne donnent une image satisfaisante de l'hétérogénéité des valeurs du CUDa de chaque acide aminé. Dans le cas du lot pois chiche, les valeurs de
CUDa oscillent seulement entre $71 \%$ pour la méthionine et $88 \%$ pour l'histidine. Dans le cas des lots fève et lentille le CUDa de l'arginine atteint les valeurs les plus élevées $(87 \%)$ alors que les valeurs les plus faibles apparaissent pour la cystine et la méthionine : 60 et $53 \%$ respectivement dans le cas de la fève et 39 et $52 \%$ dans le cas de la lentille. L'utilisation digestive apparente des acides aminés soufrés de la fève et de la lentille est particulièrement faible et le déficit de leur apport dans la ration risque donc d'en être amplifié.

Nous avons estimé l'importance quantitative de certains facteurs pouvant intervenir dans la digestion (à partir de leur teneur dans les graines de fève, lentille et pois chiche, résumée dans le tableau VI), dans la ration moyenne ingérée par les rats utilisés dans l'expérience de digestibilité.

Les proportions de cellulose ingérée atteignent respectivement $5 ; 1,6$ et $2 \%$ de la ration des lots fève, lentille et pois chiche. Parmi les constituants glucidiques des graines, l'amylose peut être considéré comme peu digestible dans la mesure sur- 

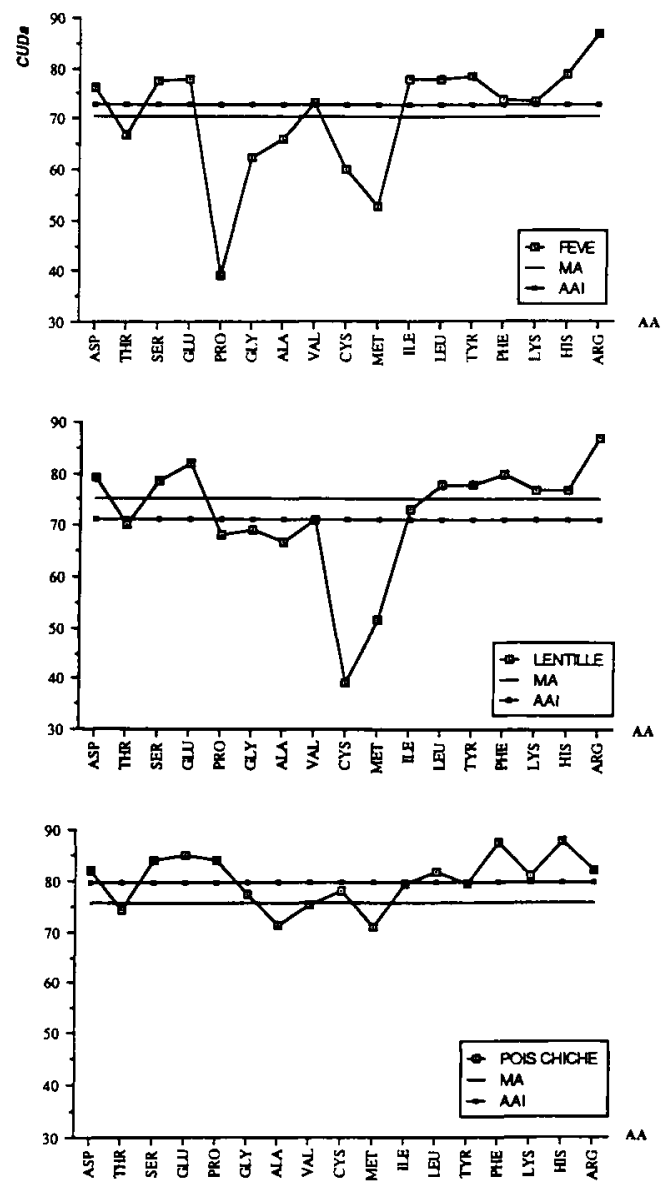

Fig 1. Comparaison du CUDa des 17 acides aminés de la fève, de la lentille et du pois chiche. Pour chaque acide aminé, le CUDa a été calculé à partir des quantités ingérées et des quantités excrétées dans les fèces.

tout où il a subi un traitement hydrothermique, mais ceci n'est que partiellement le cas dans notre étude (cf: Méthodes). On peut estimer que les proportions d'amylose de la ration sont voisines de $15 \%$ pour les lots fève et lentille mais n'atteignent pas $10 \%$ pour le lot pois chiche. Par contre, les $\alpha$-galactosides sont considérés comme de véritables facteurs antinutrition- nels et nous avons estimé à $1 ; 2,3$ et $2,6 \%$ les proportions de ces substances dans les rations des lots fève, lentille et pois chiche respectivement. Donc si la proportion du total des glucides indisgestibles dans la ration est voisine de $20 \%$ pour les lots fève et lentille, elle n'atteint pas $15 \%$ dans le cas du lot pois chiche.

Parmi les facteurs antinutritionnels, la proportion de tanins (acide tanique) atteint $3 \%$ dans le cas des lots fève et lentille et $1 \%$ pour le lot pois chiche, ce qui est inférieur au taux au-delà duquel Mitjavila et al (1978) observent un effet néfaste sur l'activité des entérocytes et l'efficacité protéique du régime. Les niveaux d'activité hémagglutinine sont très faibles pour le lot lentille et atteignent respectivement 33 et $117 \mathrm{Ui} /$ g pour les rations des lots fève et pois chiche. Le niveau d'activité des antiprotéases reste largement inférieur à $100 \mathrm{UI} / \mathrm{g}$ dans les rations des 3 lots.

\section{Utilisation métabolique}

\section{Appréciation globale}

Les valeurs du coefficient d'utilisation pratique (CUP) et du coefficient de rétention (CR) de l'azote (tableau VII) sont plus faibles dans le cas des lots ayant ingéré la fève et surtout la lentille que dans le cas du lot ayant ingéré le pois chiche.

Dans l'expérience de croissance, l'alimentation ad libitum des 3 lots de rats a permis de mettre en évidence une différence dans le niveau d'ingestion des 3 graines de légumineuses crues, mais les quantités ingérées sont toujours suffisantes pour assurer une bonne croissance. Les quantités ingérées et les vitesses de croissance des rats (tableau VIII) sont significativement $(P<0,05)$ plus élevées dans le cas de l'ingestion du pois chiche que dans le cas de l'ingestion de la 
Tableau VI. Glucides complexes et facteurs antinutritionnels des graines.

\begin{tabular}{|c|c|c|c|}
\hline & $\begin{array}{c}\text { Fève } \\
\text { Vicia faba }\end{array}$ & $\begin{array}{c}\text { Lentille } \\
\text { Lens esculenta }\end{array}$ & $\begin{array}{l}\text { Pois chiche } \\
\text { Cicer arietinium }\end{array}$ \\
\hline Glucides (\% MS) & $66^{a}$ & $68-74^{a}$ & $69 \mathrm{a} ; 65^{\mathrm{b}}$ \\
\hline $\begin{array}{l}\text { Glucides solubles } \\
\text { non amylacés }\end{array}$ & & $0,8^{c}$ & $2,1^{c}$ \\
\hline Amidon & $\begin{array}{l}40-57^{\mathrm{d}} \\
30-439 \\
41^{\mathrm{h}}\end{array}$ & $52-59^{e}$ & $39-51$ et $46-57^{\dagger}$ \\
\hline Amylose (\% amidon) & $\begin{array}{l}309 \\
34 i \\
37 i\end{array}$ & $\begin{array}{l}309 \\
40^{i}\end{array}$ & $25-42$ et $29-37^{f}$ \\
\hline Cellulose & $\begin{array}{l}7-89 \\
8,1^{k} \\
81^{1}\end{array}$ & $\begin{array}{l}3,6-4,2^{\circ} \\
4,3^{\mathrm{k}}\end{array}$ & $\begin{array}{l}7,4-2,8^{\mathrm{a}} \\
4,11^{\mathrm{b}} \\
3,2-10^{\mathrm{m}} \\
2,5-9,5^{\mathrm{m}}\end{array}$ \\
\hline Hemicellulose & $1,42^{h}$ & & $4-5^{m}$ \\
\hline $\begin{array}{l}\text { Pectines } \\
\text { Lignine } \\
\text { Oligosacharides } \\
\text { Stachyose } \\
\text { Verbascose } \\
\text { Raffinose } \\
\text { Cicéritol }\end{array}$ & $\begin{array}{l}0,80^{\circ} \\
1,08^{h} \\
4,73^{h} \\
0,67^{h} \\
1,45^{h} \\
0,22^{h}\end{array}$ & $\begin{array}{l}0,1^{p} \\
2,7^{q} \\
1,4^{r} \\
0,9^{h} \\
1,6^{r}\end{array}$ & $\begin{array}{l}0,06^{p} \\
4,04-6,48^{r} \\
0 ; 4,2^{r} \\
1-2,1^{h}\end{array}$ \\
\hline $\begin{array}{l}\text { Tanins } \\
\text { Polyphenols } \\
\text { Vicine, convicine }\end{array}$ & $\begin{array}{l}1^{d} \\
0,7^{s} \\
0,19-0,37^{t}\end{array}$ & & $\begin{array}{l}0,6-0,7 \mathrm{~m} \\
0,47-0,21^{\mathrm{s}}\end{array}$ \\
\hline $\begin{array}{l}\text { Activité (Ul/g) } \\
\text { Hemagglutinine } \\
\text { Anti-trypsique }\end{array}$ & $\begin{array}{l}4,1^{u} \\
3,2^{u} \\
11^{v}\end{array}$ & $\begin{array}{l}0,4-0,8^{v} \\
8^{v}\end{array}$ & $\begin{array}{l}16^{w} \\
10-14^{m} \\
4^{w} \\
13^{s}\end{array}$ \\
\hline Anti-chymotrypsique & & & $8^{s}$ \\
\hline
\end{tabular}

a : Duke (1981); ${ }^{\text {b }}$ : Del-Angel et Sotelo (1982); ${ }^{c}$ : Champ et al (1986); ${ }^{d}$ : Askar (1986); ${ }^{\theta}$ : Shahen et al (1978); 1 : Saini et Knights (1984); 9 : Colonna et Mercier (1979); ${ }^{n}$ : Cerning-Beroard et Filiâtre $(1976) ; 1$ : Würsch et al

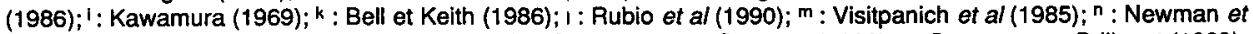
al (1987); ; : Tanusi et al (1972); $p$ : Delort-Laval et Champ (1987); $q$ : Arora (1982); $r$ : Quemener et Brillouet (1983); s: Sing et Jambunathan (1981); $t$ : Sosulski et Mccurdy (1987); $v$ : le Tien Vinh et al (1985); ${ }^{v}$ : Mansouri (1983); w: Khader et Rao (1982).

fève et surtout de la lentille. Cependant, les indices de consommation des lots fève et pois chiche ne sont pas significativement différents. Par ailleurs, les valeurs du coefficient d'efficacité protéique (CEP) des graines de lentilles et de pois chiche sont légèrement supérieures à celles des graines de fève. Cependant, si on calcule 
Tableau VII. Coefficient d'Utilisation Pratique (CUP) ot Coefficient de Rétention (CR) de l'azote.

\begin{tabular}{lccc}
\hline & Fève & Lentille & Pois chiche \\
\hline CUP & 59,2 & 54,4 & 63,6 \\
CR & 82,4 & 71,8 & 84,3 \\
\hline
\end{tabular}

$C U P=\frac{I-F-U}{I} \times 100 ; C R=\frac{1-F-U}{1-F} \times 100$

$I=$ Quantité d'azote ingérée; $F=$ Quantité d'azote excrétée dans les fèces, $U$ = Quantité d'azote excrétée dans l'urine (pour la même période).

ce coefficient en fonction de la quantité de protéines ingérées digestibles, sa valeur ne varie plus que de $1,4 \%$ entre les 3 lots.

Nous avons calculé les quantités moyennes d'acides aminés ingérées digestibles (QID) pour chacun des 3 lots utilisés dans l'expérience de croissance à partir de la composition en acides aminés des graines, de leur coefficient de digestibilité apparente, de la composition du régime et des quantités moyennes de matière sèche ingérée (MSI) pendant la période expérimentale correspondante.
Dans le cas des lots fève et pois chiche, les QID des acides aminés indispensables dépassent les besoins : 107 et $117 \%$ pour les acides aminés soufrés, 128 à $300 \%$ pour les autres. Dans le cas du lot lentille où les quantités ingérées sont plus faibles que dans les 2 autres lots, la prise en compte du coefficient de digestibilité apparente des acides aminés entraîne que l'apport de certains acides aminés indispensables est inférieur aux besoins standards : c'est le cas des acides aminés soufrés (QID = 79\% du besoin), de la lysine $(83 \%)$ et de l'histidine (86\%).

\section{Teneurs en acides aminés libres du sang et du muscle (tableau IX)}

La somme des teneurs en acides aminés libres (total AAL) du sang est voisine de 45 $\mathrm{mg} / 100 \mathrm{~g}$ pour chacun des 3 lots $(\mathrm{CV}=$ $5 \%$ ) avec une proportion un peu supérieure à $50 \%$ pour les AAl. Les concentrations en thréonine et en lysine sont assez élevées et apportent la moitié des teneurs en $\mathrm{AAl}$, les teneurs en acides aminés à chaîne ramifiée (AACR) apportent un peu moins de $20 \%$ du total AAL. L'arginine est présente en grande concentration, surtout dans le cas du lot lentille et du lot fève. Par contre, les teneurs en méthionine sont très

Tableau VIIl. Consommation et croissance des rats des 3 lots ( $\mathrm{g} / \mathrm{j} / \mathrm{animal})$.

\begin{tabular}{lccc}
\hline $\begin{array}{c}\text { Expérience } \\
\text { de croissance }\end{array}$ & Fève & Lentille & Pois chiche \\
\hline MSI & & \\
Croissance & $18,8 \pm 2,5^{\mathrm{a}}$ & $16,3 \pm 1,8^{\mathrm{b}}$ & $20,2 \pm 2,4^{\mathrm{c}}$ \\
Indice de consommation & $8,9 \pm 0,6^{\mathrm{a}}$ & $6,7 \pm 1,1^{\mathrm{b}}$ & $9,1 \pm 0,9^{\mathrm{c}}$ \\
CEP & $2,1 \pm 0,11^{\mathrm{a}}$ & $2,5 \pm 0,20^{\mathrm{b}}$ & $2,2 \pm 0,17^{\mathrm{a}}$ \\
& 2,49 & 2,74 & 2,65 \\
\hline
\end{tabular}

Moyennes \pm écart type. Les valeurs affectées d'une même lettre sur une ligne ne sont pas significativement différentes au seuil de $P<0,05$. Indice de consommation : MSI / croissance. CEP : coefficient d'efficacité protéique : croissance / quantité de (MA) protéine ingérée. 
Tableau IX. Teneurs en acides aminés libres du sang et du muscle (mg/100 g).

\begin{tabular}{|c|c|c|c|c|c|c|}
\hline & \multicolumn{2}{|c|}{ Fève } & \multicolumn{2}{|c|}{ Lentille } & \multicolumn{2}{|c|}{ Pois chiche } \\
\hline & Sang & Muscle & Sang & Muscle & Sang & Muscle \\
\hline Asp & 1,02 & 7,94 & 0,99 & 9,13 & 1,13 & 8,70 \\
\hline Thr & 5,84 & 21,23 & 5,55 & 19,83 & 4,23 & 15,66 \\
\hline Ser & 5,35 & 30,22 & 5,34 & 25,22 & 4,97 & 23,82 \\
\hline Glu & 3,40 & 51,07 & 3,04 & 36,48 & 4,25 & 46,25 \\
\hline Gly & 5,06 & 66,74 & 3,42 & 35,76 & 3,41 & 44,96 \\
\hline Ala & 5,25 & 36,93 & 5,43 & 35,50 & 4,34 & 34,76 \\
\hline Val & 1,97 & 2,33 & 1,50 & 1,97 & 1,76 & 2,37 \\
\hline Met & 0,47 & 0,31 & 0,43 & ND & 0,55 & 0,38 \\
\hline $\mathrm{\| e}$ & 1,24 & 2,88 & 0,99 & 3,29 & 1,08 & 3,20 \\
\hline Leu & 1,84 & 1,81 & 1,47 & 1,47 & 1,60 & 2,04 \\
\hline Tyr & 1,93 & 3,39 & 1,44 & 2,80 & 1,72 & 3,68 \\
\hline Phe & 0,79 & 4,14 & 0,91 & 3,77 & 0,79 & 5,77 \\
\hline Lys & 7,28 & 17,22 & 5,84 & 28,87 & 6,62 & 24,64 \\
\hline His & 1,37 & 6,25 & 1,23 & 6,67 & 1,06 & 4,80 \\
\hline Arg & 3,24 & 6,94 & 4,14 & 23,46 & 3,51 & 13,36 \\
\hline Pro & 2,05 & 4,43 & 2,21 & 2,83 & 1,64 & 3,61 \\
\hline AAl & 25,97 & 66,50 & 23,50 & 92,13 & 22,92 & 75,90 \\
\hline Total AA & 48,10 & 263,83 & 43,92 & 237,05 & 42,66 & 238,00 \\
\hline
\end{tabular}

ND : Non dosé. AAI : Acides Aminés Indispensables.

faibles, notamment dans le cas des lots lentille et fève.

Le total AAL du muscle est 5 fois plus élevé que celui du sang avec une proportion des AAl (39 à 25\%) nettement différente. La somme des teneurs musculaires en AAl est la plus faible pour les rats du lot fève, et elle est nettement plus élevée pour les rats du lot lentille que pour ceux du pois chiche. Ceci peut être attribué principalement à une forte accumulation d'arginine et de lysine libres dans le ccmpartiment musculaire des rats des lots lentille et pois chiche. En effet, le rapport des concentrations du muscle et du sang est de 5,66 pour l'arginine (et de 4,94 pour la lysine) dans le cas du lot lentille. Ce rapport est encore de 3,8 (et 3,7 ) dans le cas du lot pois chiche alors qu'il n'atteint que 2,14 (et 2,36) dans le cas du lot fève.
On observe aussi dans le cas des 3 lots étudiés une forte accumulation de phénylalanine, d'histidine $(r=5)$ et de thréonine $(r=3,6)$, ainsi qu'une certaine accumulation d'isoleucine $(r=2,32 ; 3,32 ; 2,96$ pour les lots fève, lentille et pois chiche respectivement) bien que la valeur de ce rapport (r) reste classiquement voisine de 1 pour les autres acides aminés à chaîne ramifiée.

\section{DISCUSSION}

\section{Digestibilité apparente}

Les valeurs du CUDa correspondent à celles publiées par Mendès Pereira et al, (1977); Bell et Keith (1986); Sarwar et 
Pearce (1986); Combe et al (1988). Le CUDa de l'azote peut être sous-estimé, notamment pour les graines de légumineuses, par l'importance des pertes d'azote endogène digestives. Costa de Oliviera et Sgarbieri (1986) montrent, à l'aide d'isotopes ${ }^{14} \mathrm{C}$ et ${ }^{15} \mathrm{~N}$, que les rats ayant ingéré des régimes à base de Phaseolus vulgaris excrètent significativement davantage d'azote endogène que ceux ayant ingéré le régime caséine contenant le même taux de fibre; mais leur CUD réel est significativement plus faible pour les régimes haricot que pour le régime témoin caséine.

Dans le cas d'un apport protéique sous forme de graines de légumineuses ou de céréales, il faut noter la simultanéité de l'apport des autres constituants de la graine, notamment de ses enveloppes. Laplace et al (1989) ont montré que la nature des fibres (enveloppes des graines de soja ou son de blé) avait un effet significatif sur la digestibilité iléale apparente des acides aminés chez le porc et que l'association des 2 types de fibres avait un effet négatif sur la digestibilité fécale apparente de la plupart des acides aminés. Pour expliquer les différences importantes dans la digestibilité des acides aminés des légumineuses et des céréales, Sarwar et Pearce (1986) font appel aux différences existantes dans la structure et la composition des protéines végétales. D'après les travaux de biologie moléculaire sur les graines de pois (Lycett et al, 1984), de soja (Négoro et al, 1985) et de fève (Baumlein et al, 1986) les séquences des polypeptides (et notamment la fréquence des acides aminés soufrés) constitutifs de la glycinine ou des légumines seraient régulées spécifiquement au niveau du génome. La présence des acides aminés soufrés dans les protéines de stockage est de nature à améliorer la valeur nutritionnelle protéique des graines dans la mesure où ceux-ci seront rendus disponibles en tant que nutriments. Si tel n'est pas le cas, la valeur de l'apport alimentaire est réduite par la médiocrité de sa disponibilité ou de sa digestibilité. Pour les glucides on a montré que l'amidon du pois et de la fève prenait une texture plus ferme que celle des amidons de céréales (Vose, 1980) et qu'il avait un pouvoir de gonflement plus faible que l'amidon des lentilles (Bhatty, 1988).

Les rations utilisées à base de fève, lentille ou pois chiche ne contiennent pas de concentrations élevées en tanins, facteurs hémagglutinine ou antiprotéase. Elles contiennent cependant des proportions significatives en cellulose, amylose ou en certains $\alpha$-galactosides qui favorisent l'activité microbienne dans le caecum et le côlon.

La forte teneur en cellulose de la ration fève pourrait être à l'origine de la différence de digestibilité de l'énergie constatée pour le lot fève par rapport aux deux autres lots.

Ces composés séjournent dans le caecum et le côlon des monogastriques qui ne possèdent ni cellulases ni $\alpha$-galactosidases et y sont l'objet de fermentations microbiennes. Ainsi le raffinose, le stachyose et les pentosanes ingérés en quantité élevée avec un repas à base de pois chiche entraînent une production significative d'hydrogène chez le rat, alors que l'ingestion d'un repas à base de lentille correspondant à un ingéré plus faible en sucres indigestibles n'entraîne pas de production d'hydrogène significativement différente de celle observée dans le cas de l'ingestion d'un repas caséine (Fleming, 1981). Certains des substrats énergétiques réputés non digestibles favorisent donc la croissance des bactéries. Dans le caecum et le côlon, certains produits du métabolisme bactérien pourraient éventuellement être utilisés comme nutriments énergétiques par les tissus épithéliaux. 
Le calcul des quantités moyennes d'acides aminés ingérées digestibles (QID), nous conduit à considérer que l'apport en acides aminés soufrés en lysine et histidine, peut être légèrement inférieur aux besoins du rat en croissance dans le seul cas du lot lentille. Cette conclusion ne permet pas d'expliquer les faibles valeurs du CEP des Légumineuses : en effet, dans l'expérience de croissance les valeurs les plus favorables du CEP sont enregistrées pour la lentille et pour le pois chiche. Nous avons donc voulu la confronter à un autre paramètre de l'utilisation métabolique constitué par l'équilibre du pool des acides aminés libres sanguins et musculaires.

\section{Régulation des concentrations des AAL}

L'efficacité de l'apport alimentaire des acides aminés indispensables pour les synthèses protéiques dépend de la simultanéité de leur présence sur le lieu de synthèse. La carence de l'un d'entre eux entraîne l'arrêt des synthèses et donc de I'utilisation anabolique des autres. Les concentrations en certains AAI peuvent alors augmenter. Donc, avant que l'organisme n'accuse des signes évidents de carence, les équilibres des pools d'acides aminés libres sont modifiés. L'étude de ces modifications a été proposée pour apprécier l'utilisation métabolique des protéines des aliments (Pion, 1973).

Dans le cas d'un apport alimentaire de légumineuses ajusté aux besoins, un déséquilibre éventuel dans les quantités d'acides aminés essentiels disponibles pour les synthèses protéiques peut être décelé par une accumulation sanguine et musculaire de certains acides aminés libres apportés en excès et non métabolisés, accompagnée d'une chute des acides aminés dont l'apport au niveau métabolique est insuffisant. Pion et al (1979) ont ainsi noté des teneurs sanguines et mus- culaires anormalement basses en thréonine. II en est de même pour les teneurs plasmatiques en tryptophane et méthionine des rats ayant reçu des régimes à base de lentille (ou de fève) rapportées par Sarwar et Pearce (1986).

Les teneurs sanguines en méthionine des rats des lots fève et pois chiche n'atteignent pas $50 \%$ de celles des rats des lots ayant des croissances voisines et ayant ingéré des QID comparables sous forme de farine de hareng ou de caséine (Mendès Pereira et al, 1975; Grizard et al, 1976; Dohm et al, 1981; Suzić et al, 1987). Dans le cas du lot lentille où le QID peut être inférieur aux besoins, la teneur sanguine en méthionine atteint $70 \%$ de celle d'un lot ayant ingéré un QID comparable sous forme de farine de hareng. Nous en concluons que l'apport en acides aminés soufrés des graines n'est pas complètement disponible.

En outre, dans le cas du lot lentille, l'accumulation des autres acides aminés indispensables : thréonine, lysine et arginine dans le muscle peut être provoquée par le ralentissement des synthèses protéiques dû à la faible disponibilité des acides aminés soufrés.

Dans les 2 autres lots où l'on n'a pas de risque de carence, l'accumulation de thréonine sanguine et musculaire est plus importante dans le cas du lot fève où l'ingéré digestible (QID) est cependant plus faible que dans le cas du lot pois chiche. De même, l'accumulation musculaire de lysine et d'arginine est nettement plus importante dans le cas du lot pois chiche que du lot fève où cependant les QID de lysine et d'arginine étaient voisines.

La disponibilité de la lysine et de l'arginine est donc plus élevée dans le cas de l'apport par la graine de pois chiche que par celle de fève et la disponibilité de la thréonine serait plus élevée dans le cas de l'apport par la graine de fève. 


\section{CONCLUSION}

Le déséquilibre des AAl des graines de légumineuses (défauts en cystine, méthionine, valine et thréonine et surcharge en arginine) est aggravé par leur utilisation digestive. Celle-ci semble liée à celle des polysaccharides et glucides complexes apportés simultanément par les graines et que la microflore intestinale pourrait alors utiliser pour son développement.

Les quantités ingérées digestibles (QID) des AAI dans chacun des lots sont généralement suffisantes pour satisfaire aux besoins du rat en croissance, sauf dans le cas de la lentille où ils ne sont couverts qu'à $80 \%$. L'étude des pools des acides aminés libres du sang et du muscle confirme que la méthionine est peu disponible dans les 3 espèces de graines étudiées. Elle apporte aussi des informations permettant d'avancer que la lysine et l'arginine de la fève et la thréonine du pois chiche ne seraient pas partiellement disponibles pour les synthèses protéiques des tissus périphériques.

\section{REMERCIEMENTS}

G Branlard de la station d'amélioration des plantes INRA de Crouelle a réalisé le dosage de l'acide tanique dans les graines de légumineuses. H Lafarge a compilé les références bjbliographiques à partir d'un programme d'édition élaboré par $\mathrm{D}$ Bonin. $\mathrm{M}$ Borel et $\mathrm{R}$ Tardy ont assuré la présentation du texte et des tableaux.

\section{RÉFÉRENCES}

Arora SK (1982) Légume carbohydrates. In: Chemistry and biochemistry of legumes (SK Arora, ed) Mohan Primlani, Oxford and IBM Publishing $\mathrm{Co}, 1-50$
Askar A (1986) Faba beans (Vicia faba L) and their role in the human diet. Food Nutr Bull 8 , 15-22

Baumlein $\mathrm{H}$, Wobus $\mathrm{U}$, Pustell $\mathrm{J}$, Kafatos FC (1986) The legumin gene family: structure of a B type of Vicia faba and a possible legumin gene specific regulatory element. Nucleic Acids Res 14, 2707-2720

Bhatty RS (1988) Composition and quality of lentil (Lens culinaris Medik): a review. Can Inst Food Sci Technol J 21, 144-160

Bell JM, Keith MO (1986) Nutritional and monetary evaluation of damaged lentils for growing pigs and effects of antibiotic supplements. Can J Anim Sci 66, 529-536

Carré B, Leclercq QB (1985) Digestion of polysaccharides, protein and lipids by adult cockerels fed on diets containing a pectic cell-wall material from white lupin (Lupinus albus $L$ ) cotyledon. Br J Nutr 54, 669-680

Cerning-Béroard J, Filiâtre A (1976) A comparison of the carbohydrate composition of legume seeds: horsbeans, peas, and lupin. $\mathrm{Ce}$ real Chem 53, 968-978

Champ M, Brillouet JM, Rouau X (1986) Nonstarchy polysaccharides of Phaseolus vulgaris, Lens esculenta, and Cicer arietinum seeds. J Agric Food Chem 34, 326-329

Colonna P, Mercier C (1979) Les amidons de légumineuses. Aspects, composition, structure et propriétés physico-chimiques. LebensmWiss Technol 12, 1-12

Colonna P, Guéguen J, Mercier C (1981) Pilot scale preparation of starch and cell-wall material from Pisum sativum and Vicia faba. Sci Alim 1, 415-426

Combe E, Naudin-Lebecque A, Pion R (1988) Influence de la variété et de la cuisson du pois (Pisum sativum) sur la nature des composés azotés des fèces de rats. Ann Zootech 37, 233-254

Costa de Oliviera A, Sgarbieri VC (1986) Effects of diets containing dry beans (Phaseolus vulgaris, L) on the rat excretion of endogenous nitrogen. J Nutr 116, 2387-2392

Del Angel AR, Sotelo A (1982) Nutritive value of mixtures using chick-peas with wheat, triticale, normal and Opaque-2 corns. I Nutr $112,1474-1480$

Delort-Laval J, Champ M (1987) Composition of the non-starchy polysaccharides of protein 
extraction residues from legume seeds. In: Degradation of lignocellulosics in ruminants and in industrial processes (Van der Meer JM, Rijkens BA, Ferranti MP, eds) Elsevier, Londres, 63-69

Dohm GL, Beecher GR, Warren RA (1981) Influence of exercise on free amino acid concentrations in rat tissues. $J$ App/ PHysiol $50,41-44,1981$

Duke J (1981) Handbook of legumes of world economic importance. Plenum Press, NY

El-Shobaki F, Saleh N (1986) The effect of food stuffs commonly consumed in Egypt on iron absorption and utilisation. $J$ Sci Food Agric 37, 64-68

Fleming SE (1981) Influence of cooking method on digestibility of legume and cereal starches. J Food Sci 47, 1-3

Grizard J, Maciejewska M, Prugnaud J, Pion R (1976) Influence d'une surchage en L-leucine sur les teneurs en acides aminés libres chez le rat diabétique. CR Seances Soc Biol 170 , 1, 103-111

Gupta YP (1982) Nutritive value of food legumes. In: Chemistry and biochemistry of legumes (Arora SK, ed) Mohan Primlani, Oxford and IBH Publishing $\mathrm{Co}, 287-327$

Jarrige R (1988) Alimentation des bovins, ovins, caprins. INRA, Paris

Kandé J (1967) Valeur nutritionnelle de deux graines de légumineuses : le pois chiche (Cicer arietinum) et la lentille (Lens esculenta). Ann Nutr Alim 21, 45-67

Kawamura S (1969) Studies of the starches of edible legume seeds. J Jpn Soc Starch Sci $17,19-40$

Khader V, Rao SV (1982) Limiting amino acids in Bengal gram (Cicer arietinum) as determined from blood amino acid levels and amino acid supplementation studies in the rat. $A n n$ Nutr Metab 26, 353-359

Khanvilkar SG, Desai BB (1981) Genotypic variations in protein quality and nutritional composition of chick pea (Cicer arietinum L). J Maharashtra Agric Univ 6, 226-228

Lacassagne L, Francesch M, Carré B, Melcion JP (1988) Utilization of tannin-containing and tannin-free faba bean (Vicia faba) by young chicks: effects of pelleting feeds on energy, protein and starch digestibility. Anim Feed Sci Technol 20, 59-68
Laplace JP, Darcy-Vrillon B, Perez JM, Henry Y, Giger S, Sauvant D (1989) Associative effects between two fibre sources on ileal and overall digestibilities of amino acids, energy and cell wall components in growing pigs. $\mathrm{Br}$ J Nutr 61, 75-87

Le Tien Vinh E, Dworschak E, Gal O, LindnerSzotyori K, Gergely A, Barna E (1985) Nutrient composition of an antinutritive agents in Vicia faba varieties. Elemezesi Ipar 39, 25-29

Lycett W, Croy R, Shirsat H, Boulter D (1984) The complete nucleotide sequence of a legumin gene from pea (Pisum sativum $\mathrm{L}$ ). Nucleic Acids Res 12, 4493-4506

Mansouri M (1983) Effets des traitements thermiques sur les facteurs antinutritionnels et la valeur alimentaire de 3 légumineuses. Thèse de Magister Sciences Alimentaires INA, Alger

Marquardt RR, McKirdy JA, Ward T, Campbell LD (1975) Amino acid, hemagglutinin and trypsin inhibitor levels, and proximate analyses of Faba beans (Vicia faba) and Faba bean fractions. Can J Anim Sci 55, 421-429

Mendès-Pereira E, Kunachowicz H, Pion R, Prugnaud $J(1975)$ Effet du chauffage en présence ou en absence de glucose sur l'utilisation digestive et métabolique des acides aminés de la caséine. $C R$ Séances Soc Biol $169,6,1511-1517$

Mendès-Pereira E, Pion R, Prugnaud J, Valluy MC (1977) Utilisation digestive apparente des acides aminés de quelques aliments chez le rat en croissance. Ann Biol Anim Biochim Biophys 17, 625-631

Mitjavila S, Lacombe C, Luong-Dinh C (1978) Étude comparée des effets de l'acide tannique et de l'acide tannique oxydé sur la croissance, la composition corporelle et l'utilisation biologique des aliments chez le rat. Nutr Metab 22, 8-21

Moore S, Spackmann DM, Stein WM (1958) Chromatography of amino acids on sulfonated polystyrene resins. Anal Chem 30, 11851190

Négoro T, Momma T, Fukazawa $C$ (1985) A cDNA clone encoding a glycinin Ala subunit precursor of soybean. Nucleic Acids Res 13, 6719-6731

Newman CW, Roth NJ, Newman RK, Lockerman RH (1987) Protein quality of chickpea (Cicer arietinum L). Nutr Rep Int 36, 1-8 
Paul AA, Southgate DAT (1978) The composition of foods. Mc Cance et Widdowson's $4^{\circ}$ ed

Pawlak M, Pion R (1968) Influence de la supplémentation des protéines de blé par des doses croissantes de lysine sur la teneur en acides aminés libres du sang et du muscle du rat en croissance. Ann Biol Anim Biochim Biophys 8, 517-530

Pion $A$ (1973) The relationships between the levels of free amino acids in blood and the nutritive value of proteins. In: Proteins in human nutrition (Porter JWG, Rolls BA, eds) Nato Advanced Study Institute Acad Press, Londres, 329-342

Pion R, Mendès-Pereira E, Prugnaud J (1979) Effect of composition and processing on the nutritive value of some leguminous seeds. $J$ Am Oil Chem Soc 56, 150-155

Quemener B, Brillouet JM (1983) Circitol, a pinitol digalatoside from seeds of chickpea, lentil, lupin blanc. Phytochemistry 22, 17451751

Rubio LA, Brenes A, Castano M (1990) The utilization of raw and autoclaved faba beans (Vicia faba $L$ ), var minor and faba bean fractions in diets for growing broiler chickens. $\mathrm{Br}$ J Nutr 63, 419-430

Saini HS, Knights EJ (1984) Chemical constitution of starch and oligosaccharide compartments of "Desi" and Kabuli chickpea (Cicer arietinum) seed types. I Agric Food Chem 32, 940-944

Sarwar G, Pearce RW (1986) Comparisons between true digestibility of total nitrogen and limiting amino acids in vegetable proteins fed to rats. J Nutr 116, 1172-1184

Shahen N, Roushdi M, Hassan RA (1978) Studies on lentil starch. Staerke 30, 148-150
Shekib LAH, Zoveil ME, Youssef MM, Mohamed MS (1986) Amino acid composition and in vitro digestibility of lentil and rice proteins and their mixture (Koshary). Food Chem 20, 6167

Singh U, Jambunathan $R$ (1981) Studies on desi and kabuli chickpea (Cicer arietinum $\mathrm{L}$ ) cultivars: levels of protease inhibitors, levels of polyphenolic compounds and in vitro protein digestibility. J Food Sci 46,1364-1367

Sosulski FW, McCurdy AR (1987) Functionality of flours, protein fractions and isolates from field peas and faba bean. J Food Sci 52 , 1010-1014

Suzić S, Radunovic L, Jankovic V, Segovic R (1987) Effects of protein-free diet in amino homeostatis of rat blood plasma and gut contents. FEBS Lett 216, 287-290

Tanusi S, Kawamura S, Kasait T (1972) Methods of determining carbohydrates in mature legume seeds. J Soc Food Nutr 25, 38-41

Tempel AS (1982) Tanin-measuring techniques. J Chem Ecol 8, 10, 1289-1298

Visitpanich T, Batterham ES, Norton BW (1985) Nutritional value of chickpea (Cicer arietinum) and pigeonpea (Cajanus cajan) meals for growing pigs and rats. Energy content and protein quality. Aust J Agric Res 36, 327335

Vose JR (1980) Production and functionality of starches and protein isolates from legume seeds (field peas and horsebeans). Cereal Chem 57, 406-410

Würsch P, Del Vedovo S, Koellieuter B (1986) Cell structure and starch nature as key determinants of the digestion rate of starch in legume. Am J Clin Nutr 43, 25-29 